\title{
APPLICATIONS OF EXTREME POINTS TO DISTORTION ESTIMATES
}

\author{
by
}

\author{
Johnny E. Brown
}

AbStRaCt. Sharp distortion theorems for close-to-convex functions which satisfy $\operatorname{Re} f^{\prime}(z)>\gamma$ are proved using extreme points.

\section{Introduction}

Let $\mathcal{H}(\Delta)$ be the class of all functions which are analytic in the unit disk $\Delta$. For $0 \leq \gamma<1$, we let $R_{\gamma}$ denote the subclass of functions $f \in \mathcal{H}(\Delta)$ which satisfy the condition $\operatorname{Re} f^{\prime}(z)>\gamma$ for all $z \in \Delta$, with the normalization $f(0)=0$ and $f^{\prime}(0)=1$. By the Noshiro-Warschawski-Wolff Theorem (see [2] for example), it is well-known that all functions in $R_{\gamma}$ are univalent and belong to the classical family of univalent functions $S$. In fact, functions in $R_{\gamma}$ are close-to-convex. The class $R_{0}$ has been studied and many results known ([7]). Thomas [9] proved that if $f \in R_{0}$ and $|z|=r<1$, then

$$
\left|\frac{z f^{\prime}(z)}{f(z)}\right| \leq \frac{K}{-(1-r) \log (1-r)}
$$

and stated that extreme point methods did not seem to give sharp bounds. Later, London [6] found the sharp bound without using extreme points. The purpose of this paper is to show that extreme point methods can be used to both generalize London's result for all $0 \leq \gamma<1$ and give sharp estimates for other distortion results. The basic method given here may be used in other types of extremal problems for other families of functions.

\section{Main Results}

For each $0 \leq \gamma<1$, for convenience only, we henceforth set

$$
\alpha=1-2 \gamma
$$

and define functions $k_{\alpha} \in R_{\gamma}$ as follows :

$$
k_{\alpha}(z)=-\alpha z-(1+\alpha) \log (1-z) .
$$

These and their rotations will turn out to be extremal functions. We can now state our results.

1991 Mathematics Subject Classification. Primary 30C45; Secondary 30C75.

Key words and phrases. Close-to-convex functions, distortion estimates, extreme points. 
Theorem 2.1. If $f \in R_{\gamma}, 0 \leq \gamma<1$ and $|z|=r<1$, then

$$
\frac{\left|f^{\prime}(z)\right|}{\operatorname{Re}\left\{\frac{f(z)}{z}\right\}} \leq \frac{r k_{\alpha}^{\prime}(r)}{k_{\alpha}(r)}=\frac{r(1+\alpha r)}{(1-r)[-\alpha r-(1+\alpha) \log (1-r)]} .
$$

Equality holds for $0<r<1$ when $f(z)=k_{\alpha}(z)$.

The above result also gives the sharp upper bound for both $\left|z f^{\prime}(z) / f(z)\right|$ and for $\operatorname{Re}\left\{f^{\prime}(z)\right\} / \operatorname{Re}\{f(z) / z\}$.

Theorem 2.2. If $f \in R_{\gamma}, 0 \leq \gamma<1$ and $|z|=r<1$, then

$$
\frac{\left|z f^{\prime \prime}(z)\right|}{R e\left\{f^{\prime}(z)\right\}} \leq \frac{r k_{\alpha}^{\prime \prime}(r)}{k_{\alpha}^{\prime}(r)}=\frac{r(1+\alpha)}{(1-r)(1+\alpha r)} .
$$

Equality holds for $0<r<1$ when $f(z)=k_{\alpha}(z)$.

It is known (see [1] or[5]) that if $\operatorname{Re} f^{\prime}(z)>\gamma$, then $\operatorname{Re}\{f(z) / z\}>\gamma$. However, the sharp estimate is given by :

Theorem 2.3. If $f \in R_{\gamma}, 0 \leq \gamma<1$ and $|z|=r<1$, then

$$
\operatorname{Re}\left\{\frac{f(z)}{z}\right\} \geq-\frac{k_{\alpha}(-r)}{r}=-\alpha+\frac{(1+\alpha)}{r} \log (1+r) .
$$

Equality holds for $0<r<1$ when $f(z)=-k_{\alpha}(-z)$.

\section{Proofs}

There are some elementary properties of the class $R_{\gamma}$ which we now state. It is clear that $R_{\gamma}$ is a compact family in $\mathcal{H}(\Delta)$ (endowed with the topology of uniform convergence on compacta in $\Delta$ ). It is a convex family, i.e., if $f, g \in R_{\gamma}$, then $t f+(1-t) g \in R_{\gamma}$ for all $t \in[0,1]$. This class is also rotationally invariant $: f \in R_{\gamma}$ if and only if $e^{-i \nu} f\left(e^{i \nu} z\right) \in R_{\gamma}$ for all $\nu \in \mathbb{R}$. Observe that the derivatives of functions in $R_{\gamma}$ are related to functions in the classical Carathéordory class $\mathcal{P}$ by $f \in R_{\gamma}$ if and only if $\left(f^{\prime}-\gamma\right) /(1-\gamma) \in \mathcal{P}$. From this and the well-known fact that the extreme points of $\mathcal{P}$ are given by

$$
\mathcal{E}(\mathcal{P})=\left\{\frac{1+e^{i \mu} z}{1-e^{i \mu} z}: 0 \leq \mu \leq 2 \pi\right\}
$$

it follows that the extreme points of $R_{\gamma}$ are

$$
\mathcal{E}\left(\mathcal{R}_{\gamma}\right)=\left\{e^{-i \mu} k_{\alpha}\left(e^{i \mu} z\right): 0 \leq \mu \leq 2 \pi\right\}
$$

We need two preliminary results. The first may be compared here with that of Ruscheweyh [8]. It is the convexity of certain functionals which will be important to prove our results. (Recall that a functional $\Phi$ is convex if $\Phi(t f+(1-t) g) \leq$ $t \Phi(f)+(1-t) \Phi(g)$ for $f, g \in \mathcal{H}(\Delta)$ and all $t \in[0,1]$.) The second contains the computations necessary to complete our proofs. 
Lemma 1. Let $\mathcal{F}$ be a compact and convex family of functions in $\mathcal{H}(\Delta)$. If $\Phi$ and $L$ are real-valued continuous functionals on $\mathcal{H}(\Delta)$ which are convex and linear, respectively, with $L(\mathcal{F})>0$ and $|\Phi(\mathcal{F})|<\infty$, then

$$
\max _{f \in \mathcal{F}} \frac{\Phi(f)}{L(f)}=\max _{f \in \mathcal{E}(\mathcal{F})} \frac{\Phi(f)}{L(f)} .
$$

Lemma 2. If $J(t, x) \equiv 1+t^{2}-2 t x$ and $0<r<1$, then

(i) $\int_{0}^{r} \frac{\rho\left(1-\rho^{2}\right)}{J(\rho, x)^{2}} d \rho \leq \frac{r^{2}}{J(r, x)},-1 \leq x \leq 1$

(ii) $\int_{0}^{r} \frac{\rho\left(1-\rho^{2}\right)}{J(\rho, x)^{2}} d \rho \leq\left[\frac{r\left(1+r^{2}\right)}{J(r,-x) J(r, x)}\right] \int_{0}^{r} \frac{\left(1-\rho^{2}\right)}{J(\rho, x)} d \rho,-1 \leq x \leq 1$

(iii) $\int_{0}^{r} \frac{\rho\left(1-\rho^{2}\right)}{J(\rho, x)^{2}} d \rho \leq\left[\frac{r}{J(r, x)}\right] \int_{0}^{r} \frac{(1-\rho x)}{J(\rho, x)} d \rho,-1 \leq x \leq 1$

(iv) $\int_{0}^{r} \frac{\left(\rho x-\rho^{2}\right)}{J(\rho, x)} d \rho \leq\left[\frac{1+\alpha r^{2}}{J(\alpha r,-x)}\right] \int_{0}^{r} \frac{(1-\rho x)+\alpha\left(\rho x-\rho^{2}\right)}{J(\rho, x)} d \rho, 0 \leq \alpha, x \leq 1$.

We proceed now with the proofs of the main results. The lemmas are proved at the end of this section.

Proof of Theorem 2.1. Since $R_{\gamma}$ is rotationally invariant, it suffices to prove the inequality for $z=r$. Thus define $\Phi(f) \equiv\left|f^{\prime}(r)\right|$ and $L(f) \equiv \operatorname{Re}\{f(r) / r\}$. Note that $L\left(R_{\gamma}\right)>\gamma$. Applying Lemma 1, using (2.2) and (3.1), we conclude that for every $f \in R_{\gamma}$,

$$
\frac{\left|f^{\prime}(r)\right|}{\operatorname{Re}\left\{\frac{f(r)}{r}\right\}} \leq \max _{0 \leq \theta \leq 2 \pi} F(\theta)
$$

where

$$
F(\theta)=\frac{\left|\frac{1+\alpha r e^{i \theta}}{1-r e^{i \theta}}\right|}{\operatorname{Re}\left\{-\alpha-\left(r e^{i \theta}\right)^{-1}(1+\alpha) \log \left(1-r e^{i \theta}\right)\right\}} .
$$

By symmetry, it is without loss of generality to suppose that $0 \leq \theta \leq \pi$. The result is proved if we show that $F(\theta) \leq F(0)$.

If we let $J(t, x)=1+t^{2}-2 t x$, where $x=\cos \theta$, then

$$
\operatorname{Re}\left\{-\alpha-\frac{(1+\alpha)}{r e^{i \theta}} \log \left(1-r e^{i \theta}\right)\right\}=\frac{1}{r} \int_{0}^{r} \frac{(1-\rho x)+\alpha\left(\rho x-\rho^{2}\right)}{J(\rho, x)} d \rho
$$


and

$$
\frac{\partial}{\partial \theta} \operatorname{Re}\left\{-\alpha-\frac{(1+\alpha)}{r e^{i \theta}} \log \left(1-r e^{i \theta}\right)\right\}=-\frac{(1+\alpha)}{r}(\sin \theta) \int_{0}^{r} \frac{\rho\left(1-\rho^{2}\right)}{J(\rho, x)^{2}} d \rho
$$

and also

$$
\frac{\partial\left|\frac{1+\alpha r e^{i \theta}}{1-r e^{i \theta}}\right|}{\partial \theta}=-\frac{r(1+\alpha)\left(1+\alpha r^{2}\right) \sin \theta}{J(\alpha r,-x) J(r, x)}\left|\frac{1+\alpha r e^{i \theta}}{1-r e^{i \theta}}\right| .
$$

It follows that

$$
F^{\prime}(\theta)=\frac{(1+\alpha)}{r\left(\operatorname{Re}\left\{k_{\alpha}\left(r e^{i \theta}\right) /\left(r e^{i \theta}\right)\right\}\right)^{2}}(\sin \theta)\left|\frac{1+\alpha r e^{i \theta}}{1-r e^{i \theta}}\right| H(x),
$$

where

$$
H(x)=\left[\int_{0}^{r} \frac{\rho\left(1-\rho^{2}\right)}{J(\rho, x)^{2}} d \rho\right]-\left[\frac{r\left(1+\alpha r^{2}\right)}{J(\alpha r,-x) J(r, x)}\right] \int_{0}^{r} \frac{(1-\rho x)+\alpha\left(\rho x-\rho^{2}\right)}{J(\rho, x)} d \rho,
$$

with $x=\cos \theta$ and $0 \leq \theta \leq \pi$. It is now necessary to consider cases.

Case $1: 0 \leq \alpha \leq 1$ and $-1 \leq x \leq 1$. From the above, it is enough to show that $H(x) \leq 0$, or equivalently $\{J(\alpha r,-x) J(r, x) / r\} H(x) \leq 0$, which is true if and only if $G(\alpha) \leq 0$, where

$$
G(\alpha)=A \alpha^{2}+B \alpha+C
$$

and

$$
\begin{aligned}
& A=r^{2}\left[\frac{J(r, x)}{r} I-\int_{0}^{r} \frac{\rho x-\rho^{2}}{J(\rho, x)} d \rho\right] \\
& B=2 x J(r, x) I-\int_{0}^{r} \frac{r^{2}(1-\rho x)+\left(\rho x-\rho^{2}\right)}{J(\rho, x)} d \rho \\
& C=\frac{J(r, x)}{r} I-\int_{0}^{r} \frac{(1-\rho x)}{J(\rho, x)} d \rho
\end{aligned}
$$

with $I=\int_{0}^{r} \frac{\rho\left(1-\rho^{2}\right)}{J(\rho, x)^{2}} d \rho$. By Lemma $2(i i)$ and $(i i i)$, respectively, we get $G(1) \leq 0$ and $G(0) \leq 0$. Hence if $A \geq 0$, we have $G(\alpha) \leq 0$ and so $H(x) \leq 0$. Hence suppose $A<0$, that is

$$
\frac{J(r, x)}{r} I<\int_{0}^{r} \frac{\rho x-\rho^{2}}{J(\rho, x)} d \rho .
$$

In order for (3.6) to hold, note that we must necessarily have $0 \leq x \leq 1$. Hence to show that $H(x) \leq 0$, using (3.6) we need only verify if $0 \leq x \leq 1$ and $0 \leq \alpha \leq 1$, then

$$
\int_{0}^{r} \frac{\rho x-\rho^{2}}{J(\rho, x)} d \rho \leq\left(\frac{1+\alpha r^{2}}{J(\alpha r,-x)}\right) \int_{0}^{r} \frac{(1-\rho x)+\alpha\left(\rho x-\rho^{2}\right)}{J(\rho, x)} d \rho .
$$

This is Lemma $2(i v)$. Hence $H(x) \leq 0, F^{\prime}(\theta) \leq 0$ and so $F(\theta) \leq F(0)$. 
Case $2:-1<\alpha \leq 0$ and $\frac{r(1-\alpha)}{2} \leq x \leq 1$. In this case we must have

$$
\frac{1+\alpha r^{2}}{J(\alpha r,-x)} \geq 1
$$

and hence $H(x) \leq 0$ (and also $F^{\prime}(\theta) \leq 0$ ) follows if we show that

$$
\int_{0}^{r} \frac{\rho\left(1-\rho^{2}\right)}{J(\rho, x)^{2}} d \rho \leq \frac{r}{J(r, x)} \int_{0}^{r} \frac{(1-\rho x)+\alpha\left(\rho x-\rho^{2}\right)}{J(\rho, x)} d \rho \equiv M(\alpha) .
$$

Since $M$ is linear in $\alpha$, the above inequality holds by Lemma $2(i)$ and $(i i i)$.

Case $3:-1<\alpha \leq 0$ and $-1 \leq x \leq \frac{r(1-\alpha)}{2}$. In this situation, we see that

$$
\left|\frac{1+\alpha r e^{i \theta}}{1-r e^{i \theta}}\right| \leq 1
$$

and so the expression

$$
\left|\frac{1+\alpha r e^{i \theta}}{1-r e^{i \theta}}\right|\left\{-\alpha-\frac{(1+\alpha)}{r} \log (1-r)\right\}-\left(\frac{1+\alpha r}{1-r}\right) \operatorname{Re}\left\{-\alpha-\frac{(1+\alpha)}{r e^{i \theta}} \log \left(1-r e^{i \theta}\right)\right\}
$$

is bounded above by $H(\alpha, \theta)$, where

$H(\alpha, \theta)=\left\{-\alpha-\frac{(1+\alpha)}{r} \log (1-r)\right\}-\left(\frac{1+\alpha r}{1-r}\right) \operatorname{Re}\left\{-\alpha-\frac{(1+\alpha)}{r e^{i \theta}} \log \left(1-r e^{i \theta}\right)\right\}$.

We can show directly that $H(\alpha, \theta) \leq 0$, which is equivalent to $F(\theta) \leq F(0)$. For convenience, set $\mu(\theta)=\operatorname{Re}\left\{\left(r e^{i \theta}\right)^{-1} \log \left(1-r e^{i \theta}\right)\right\}$. Because $\frac{1}{z} \log (1-z)$ is a convex univalent function which maps disks $|z| \leq r$ onto convex regions symmetric with respect to the real axis, we get

$$
\operatorname{Re}\left\{\left(r e^{i \theta}\right)^{-1} \log \left(1-r e^{i \theta}\right)\right\} \leq-\frac{1}{r} \log (1+r) .
$$

Now we have

$$
\frac{\partial H}{\partial \alpha}=\frac{r(1+2 \alpha)}{1-r}-\frac{1}{r} \log (1-r)+\mu\left[\frac{1+(1+2 \alpha) r}{1-r}\right] .
$$

This function is linear in $\mu$ and (3.7) gives $\mu(\theta) \leq-\frac{1}{r} \log (1+r)$ and hence

$$
r(1-r) \frac{\partial H}{\partial \alpha} \leq r^{2}(1+2 \alpha)-(1-r) \log (1-r)-[1+(1+2 \alpha) r] \log (1+r) .
$$

The right hand side is linear in $\alpha$ so a brief calculation then gives $\frac{\partial H}{\partial \alpha} \leq 0$. We are done because $H(\alpha, \theta)<H(-1, \theta)=0$.

Proof of Theorem 2.2. As in the proof of the previous theorem, it suffices to prove our result for $z=r$. Put $\Phi(f) \equiv\left|r f^{\prime \prime}(r)\right|, L(f) \equiv \operatorname{Re}\left\{f^{\prime}(r)\right\}$ and apply Lemma 1 with (3.1) to conclude that for any $f \in R_{\gamma}$, 


$$
\frac{\left|r f^{\prime \prime}(r)\right|}{\operatorname{Re}\left\{f^{\prime}(r)\right\}} \leq \max _{0 \leq \theta \leq 2 \pi} K(\theta)
$$

where

$$
K(\theta)=\frac{r(1+\alpha)}{\left|1-r e^{i \theta}\right|^{2}} / \operatorname{Re}\left\{\frac{1+\alpha r e^{i \theta}}{1-r e^{i \theta}}\right\}
$$

We wish to show that $K(\theta) \leq K(0)$, but this is equivalent to

$$
(1-r)(1+\alpha r)-\left|1-r e^{i \theta}\right|^{2} \operatorname{Re}\left\{\frac{1+\alpha r e^{i \theta}}{1-r e^{i \theta}}\right\} \leq 0
$$

This in turn is equivalent to $(1-\alpha)(\cos \theta-1) \leq 0$. The proof of the theorem is complete.

Proof of Theorem 2.3. We prove this result for $z=r$. Here we let $\Phi(f) \equiv 1$ and $L(f) \equiv \operatorname{Re}\{f(r) / r\}$. From Lemma 1 and (3.1) we conclude that for any $f \in R_{\gamma}$,

$$
\operatorname{Re}\left\{\frac{f(r)}{r}\right\} \geq \min _{0 \leq \theta \leq 2 \pi} N(\theta)
$$

where $N(\theta) \equiv \operatorname{Re}\left\{k_{\alpha}\left(r e^{i \theta}\right) /\left(r e^{i \theta}\right)\right\}$. We need only prove that $N(\theta) \geq N(\pi)$, which is equivalent to $\operatorname{Re}\left\{\left(r e^{i \theta}\right)^{-1} \log \left(1-r e^{i \theta}\right)\right\} \leq-\frac{1}{r} \log (1+r)$. This is (3.7).

We now turn our attention to the proofs of the lemmas. A very useful result due to Hallenbeck and MacGregor[4,p45] is as follows:

Proposition. Let $\mathcal{F}$ be a compact family in $\mathcal{H}(\Delta)$. If $J$ is a real-valued continuous convex functional on $C H(\mathcal{F})$, the closed convex hull of $\mathcal{F}$, then

$$
\max _{f \in \mathcal{F}} J(f)=\max _{f \in \mathcal{E}(C H(\mathcal{F}))} J(f) .
$$

Proof of Lemma 1 . Since $\mathcal{F}$ is compact, there always exists an extremal function $g \in \mathcal{F}$ such that

$$
M=\sup _{f \in \mathcal{F}} \frac{\Phi(f)}{L(f)}=\frac{\Phi(g)}{L(g)} .
$$

(By hypothesis $M$ is finite.) If we let $J(f) \equiv \Phi(f)-M L(f)$, then $J$ is a continuous real-valued convex functional whose maximum on $\mathcal{F}$ is zero. In our case $\mathcal{F}$ is a convex family hence by the above Proposition there exists $g^{*} \in \mathcal{E}(\mathcal{F})$ such that $J\left(g^{*}\right)=0$, i.e., for any $f \in \mathcal{F}$,

$$
\frac{\Phi(f)}{L(f)} \leq M=\frac{\Phi\left(g^{*}\right)}{L\left(g^{*}\right)}
$$

The proof of this lemma is complete. 
Proof of Lemma 2. We consider each inequality separately.

$(i)$ : This inequality holds if $\int_{0}^{r} \frac{\partial}{\partial t}\{G(t, x)\} d t \leq 0$, where

$$
G(t, x)=\int_{0}^{t} \frac{\rho\left(1-\rho^{2}\right)}{J(\rho, x)^{2}} d \rho-\frac{t^{2}}{J(t, x)} .
$$

But clearly we see that $\frac{\partial G}{\partial t}=-t / J(t, x) \leq 0$, so $(i)$ is proved.

(ii): This inequality is proved in London [6,p523].

(iii): This inequality is true if $\int_{0}^{r} \frac{\partial}{\partial t}\{G(t, x)\} d t \leq 0$, where

$$
G(t, x)=\int_{0}^{t} \frac{\rho\left(1-\rho^{2}\right)}{J(\rho, x)^{2}} d \rho-\frac{t}{J(t, x)} \int_{0}^{t} \frac{1-\rho x}{J(\rho, x)} d \rho .
$$

It follows that

$$
J^{2}(t, x) \frac{\partial G}{\partial t}=-\left(1-t^{2}\right) \int_{0}^{t} \frac{1-\rho x}{J(\rho, x)} d \rho+t^{2}(x-t) .
$$

If $-1 \leq x \leq 0$, then $\frac{\partial G}{\partial t} \leq 0$ and so (iii) holds in this case.

Suppose now that we have $0 \leq x \leq 1$. By $(i i)$, to prove (iii) we need only verify that

$$
\frac{1+r^{2}}{J(r,-x)} \int_{0}^{r} \frac{1-\rho^{2}}{J(\rho, x)} d \rho \leq \int_{0}^{r} \frac{1-\rho x}{J(\rho, x)} d \rho
$$

which is true if and only if

$$
\int_{0}^{r} \frac{\Psi(x, \rho)}{J(\rho, x)} d \rho \leq 0
$$

where

$$
\Psi(x, \rho)=\left(1+r^{2}\right)\left(1-\rho^{2}\right)+(\rho x-1) J(r,-x)=A x^{2}+B x+C .
$$

Now clearly $A=2 \rho r \geq 0, \Psi(0, \rho)=-\rho^{2}\left(1+r^{2}\right)$, and, since $0 \leq \rho \leq r$, we get $\Psi(1, \rho)=(1-\rho)\left(\rho+r^{2} \rho-2 r\right) \leq(1-\rho)\left(r^{3}-r\right)$. Hence $\Psi(x, \rho) \leq 0$ for $0 \leq x \leq 1$, so (3.11) and (3.10) hold and thus (iii) holds.

(iv) : After multiplying both sides by $J(\alpha r,-x)$, we see that this inequality holds if and only if $K(\alpha, x) \leq 0$, where

$$
K(\alpha, x)=\int_{0}^{r} \frac{\alpha\left[(2 r x-1)\left(\rho x-\rho^{2}\right)-r^{2}(1-\rho x)\right]-J(\rho, x)}{J(\rho, x)} d \rho .
$$

Clearly $K(0, x) \leq 0$ and $K(1, x)=\int_{0}^{r} \frac{\omega(x)}{J(\rho, x)} d \rho$, where

$$
\omega(x)=(2 r x-1)\left(\rho x-\rho^{2}\right)-r^{2}(1-\rho x)-J(\rho, x)=A x^{2}+B x+C,
$$

with $A=2 \rho r \geq 0, \omega(0)=-\left(1+r^{2}\right)$ and $\omega(1) \leq(1-\rho)\left(r^{2}-1\right)$. Hence we get $K(1, x) \leq 0$ and since $K$ is linear in $\alpha$, this proves $K(\alpha, x) \leq 0$ and $(i v)$ is proved. 


\section{Remarks}

(1) By tracing through the cases of equality, we have shown that equality in all our main results are in fact achieved only for the indicated extremal functions.

(2) It is essential that we separate cases in the proof of Theorem 2.1 since $F^{\prime}(\theta) \leq 0$ is false for all $0 \leq \theta \leq \pi$ when $-1<\alpha<0$. For example, if $\alpha=-0.8, r=0.8$ and $\theta_{0}=1.4266$ then $F^{\prime}\left(\theta_{0}\right)>0$. Also, we note that cases $(i i)$ and $($ iii $)$ are completely independent of each other.

(3) Theorem 2.1 is a rather curious result since both $\left|f^{\prime}(z)\right|$ and $\operatorname{Re}\{f(z) / z\}$ are maximized for $k_{\alpha}(z)$ (and both minimized for $-k_{\alpha}(-z)$ ), while their quotient $\left|f^{\prime}(z)\right| / \operatorname{Re}\{f(z) / z\}$ is maximized by the single function $k_{\alpha}(z)$ for each $0 \leq \gamma<1$. This is not always true for estimates of other such functionals over $R_{\gamma}$. Indeed, it is of interest to observe that for fixed $z \in \Delta$,

$$
\max _{f \in R_{\gamma}} \frac{\operatorname{Re}\{f(z) / z\}}{\operatorname{Re}\left\{f^{\prime}(z)\right\}}
$$

is not always attained for the single function $-k_{\alpha}(-z)$ (or $k_{\alpha}(z)$ ) for all $0 \leq \gamma<1$. Upon selecting $z=0.85, \alpha=0$ (i.e., $\gamma=\frac{1}{2}$ ) and $\mu=\frac{\pi}{2}$, a numerical check shows that

$$
\frac{\operatorname{Re}\left\{k_{\alpha}(r) / r\right\}}{\operatorname{Re}\left\{k_{\alpha}^{\prime}(r)\right\}}<\frac{\operatorname{Re}\left\{k_{\alpha}(-r) /(-r)\right\}}{\operatorname{Re}\left\{k_{\alpha}^{\prime}(-r)\right\}}<\frac{\operatorname{Re}\left\{k_{\alpha}\left(r e^{i \mu}\right) /\left(r e^{i \mu}\right)\right\}}{\operatorname{Re}\left\{k_{\alpha}^{\prime}\left(r e^{i \mu}\right)\right\}}
$$

Thus by Theorem 2.1, the maximum of the the functional $\operatorname{Re}\left\{f^{\prime}(z)\right\} / \operatorname{Re}\{f(z) / z\}$ is attained for $f(z)=k_{\alpha}(z)$ while the minimum is not attained (for all $0 \leq \gamma<1)$ for $f(z)=-k_{\alpha}(-z)$ (or $k_{\alpha}(z)$ ) as one might expect.

(4) Theorem 2.3 was proved earlier by Hallenbeck[3] via subordination methods. Subordination methods and extreme point methods complement each other in considering extremal problems. For example subordination can be used to show that for all $f \in R_{\gamma}$,

$$
\int_{0}^{2 \pi}\left|f^{\prime}\left(r e^{i \theta}\right)\right|^{p} d \theta \leq \int_{0}^{2 \pi}\left|k_{\alpha}^{\prime}\left(r e^{i \theta}\right)\right|^{p} d \theta, \quad 0<p<\infty
$$

while extreme point methods can be used to obtain

$$
\int_{0}^{2 \pi}\left|f\left(r e^{i \theta}\right)\right|^{p} d \theta \leq \int_{0}^{2 \pi}\left|k_{\alpha}\left(r e^{i \theta}\right)\right|^{p} d \theta, \quad 1 \leq p<\infty
$$

\section{REFERENCES}

1. J. E. Brown, Quasiconformal extensions for some geometric subclasses of univalent functions, Internat. J. Math. and Math. Sci. 7(1) (1984), 187-195.

2. P.L. Duren,, Univalent Functions, Springer-Verlag Berlin and New York (1983). 
3. D. J. Hallenbeck, Convex hulls and extreme points of some families of univalent functions, Trans. Amer. Math. Soc. 192 (1974), 285-292.

4. D. J. Hallenbeck and T.H MacGregor, Linear Problems and Convexity Techniques in Geometric Function Theory, Monographs and Studies in Mathematics 22, Pitman (1984).

5. R. J. Libera, Some classes of regular univalent functions, Proc. Amer. Math. Soc. 16 (1965), 755-758.

6. R. R. London, Functions whose derivative has positive real part, Proc. Amer. Math. Soc 103 (1988), 521-524.

7. T. H. MacGregor, Functions whose derivative has a positive real part, Trans. Amer. Math. Soc. 104 (1962), 532-537.

8. S. Ruscheweyh, Nichtlineare Extremalprobleme fur holomorphe Stieltjesintegrale, Math. Z. 142 (1975), 19-23.

9. D. K. Thomas, On functions whose derivative has positive real part, Proc. Amer. Math. Soc 98 (1986), 68-70.

\author{
DEPARTMENT OF MATHEMATICS \\ PURDUE UNIVERSITY \\ WEST LAFAYETTE, IN 47907 \\ jeb@math.purdue.edu
}

